\title{
Incidental durotomy: predictive risk model and external validation of natural language process identification algorithm
}

\author{
*Jeff Ehresman, BS,' Zach Pennington, BS, ${ }^{1}$ Aditya V. Karhade, BE, ${ }^{2}$ Sakibul Huq, BS, ${ }^{1}$ \\ Ravi Medikonda, BA, ${ }^{1}$ Andrew Schilling, AB, ${ }^{1}$ James Feghali, MD, ${ }^{1}$ Andrew Hersh, BS, ${ }^{1}$ \\ A. Karim Ahmed, BS, ${ }^{1}$ Ethan Cottrill, BS, ${ }^{1}$ Daniel Lubelski, MD, ${ }^{1}$ Erick M. Westbroek, MD, ${ }^{1}$ \\ Joseph H. Schwab, MD, MS, ${ }^{2}$ and Daniel M. Sciubba, MD'
}

\begin{abstract}
'Department of Neurosurgery, Johns Hopkins University School of Medicine, Baltimore, Maryland; and 2Department of Orthopaedic Surgery, Harvard Medical School, Boston, Massachusetts
\end{abstract}

\begin{abstract}
OBJECTIVE Incidental durotomy is a common complication of elective lumbar spine surgery seen in up to $11 \%$ of cases. Prior studies have suggested patient age and body habitus along with a history of prior surgery as being associated with an increased risk of dural tear. To date, no calculator has been developed for quantifying risk. Here, the authors' aim was to identify independent predictors of incidental durotomy, present a novel predictive calculator, and externally validate a novel method to identify incidental durotomies using natural language processing (NLP).
\end{abstract}

METHODS The authors retrospectively reviewed all patients who underwent elective lumbar spine procedures at a tertiary academic hospital for degenerative pathologies between July 2016 and November 2018. Data were collected regarding surgical details, patient demographic information, and patient medical comorbidities. The primary outcome was incidental durotomy, which was identified both through manual extraction and the NLP algorithm. Multivariable logistic regression was used to identify independent predictors of incidental durotomy. Bootstrapping was then employed to estimate optimism in the model, which was corrected for; this model was converted to a calculator and deployed online.

RESULTS Of the 1279 elective lumbar surgery patients included in this study, incidental durotomy occurred in 108 $(8.4 \%)$. Risk factors for incidental durotomy on multivariable logistic regression were increased surgical duration, older age, revision versus index surgery, and case starts after 4 PM. This model had an area under curve (AUC) of 0.73 in predicting incidental durotomies. The previously established NLP method was used to identify cases of incidental durotomy, of which it demonstrated excellent discrimination (AUC 0.97).

CONCLUSIONS Using multivariable analysis, the authors found that increased surgical duration, older patient age, cases started after 4 PM, and a history of prior spine surgery are all independent positive predictors of incidental durotomy in patients undergoing elective lumbar surgery. Additionally, the authors put forth the first version of a clinical calculator for durotomy risk that could be used prospectively by spine surgeons when counseling patients about their surgical risk. Lastly, the authors presented an external validation of an NLP algorithm used to identify incidental durotomies through the review of free-text operative notes. The authors believe that these tools can aid clinicians and researchers in their efforts to prevent this costly complication in spine surgery.

https://thejns.org/doi/abs/10.3171/2020.2.SPINE20127

KEYWORDS incidental durotomy; intraoperative complication; lumbar spine surgery; natural language processing

$\mathrm{I}$ NCIDENTAL durotomy-defined by compromise of the thecal sac with or without CSF leakage-is a relatively common complication of lumbar spine surgery. Previous reports have documented incidental durotomy to occur at rates varying from $0.3 \%$ for lumbar discectomy ${ }^{1}$ to $11 \%$ for operations to correct adult spinal deformity. ${ }^{2}$ Consequences of dural tears include postoperative headache, meningitis, new-onset neurological deficit, pseudomeningocele, need for surgical revision, and significant financial costs to the patient and health system. ${ }^{3-6}$ Previous

ABBREVIATIONS ASA = American Society of Anesthesiologists; ASD-S = adult spinal deformity-surgical; $\mathrm{AUC}=$ area under the curve; $\mathrm{CCI}=\mathrm{Charlson}$ Comorbidity Index; $\mathrm{LOS}=$ length of stay; NLP = natural language processing; PRC = precision-recall curve; $\mathrm{ROC}=$ receiver operating characteristic.

SUBMITTED January 27, 2020. ACCEPTED February 21, 2020.

INCLUDE WHEN CITING Published online May 1, 2020; DOI: 10.3171/2020.2.SPINE20127.

* J.E. and Z.P. contributed equally to this work. 
investigations for risk factors of durotomy have identified revision (vs index) surgery, patient age, obesity, a medical history of diabetes mellitus, and surgical invasiveness. ${ }^{1,7}$ However, none of these prior results have been converted into an online predictive tool that can be used by spine surgeons for the purposes of preoperative patient consultation, which would have obvious advantages for the average practicing spine surgeon.

Furthermore, while the retrospective identification of the incidental durotomies may seem simple and straightforward, a recent study by Karhade et al. described how previous large database studies that identified durotomies using procedural and diagnosis codes have reported much lower incidence rates than the existing literature. ${ }^{8-10}$ It was hypothesized that use of these codes may lead to missed cases of incidental durotomy and subsequent underreporting of the true incidence. This group then created an automated natural language processing (NLP) algorithm to detect incidental durotomies based on free-text operative notes and found this method to more accurately detect durotomies compared with procedural/diagnosis codes. ${ }^{9}$ Therefore, in addition to our aim of creating a web-based calculator to predict risk of incidental durotomies, we also sought to externally validate this NLP algorithm using our institution's relatively large cohort of elective lumbar surgery patients.

\section{Methods}

After obtaining institutional review board approval, we retrospectively reviewed the medical records for all patients who underwent elective lumbar or thoracolumbar spine operations between July 1, 2016, and November 30, 2018, for degenerative pathologies. Patients were initially identified using the operative schedule in the electronic medical record to screen for procedures. Individual files were then reviewed for surgical indication and to determine whether patients met the inclusion and exclusion criteria. Inclusion criteria were that the patient underwent open surgery between T11 and the sacrum, was at least 18 years of age, had a minimum of 30-day follow-up, and had full anesthesia records and operative notes. Patients were excluded if they underwent surgery for a nondegenerative pathology (e.g., tumor, infection, congenital deformity, trauma) or had incomplete medical records. Additionally, the records of included patients were reviewed to extract details about patient demographics (age, sex), medical comorbidities (Charlson Comorbidity Index [CCI], American Society of Anesthesiologists [ASA] physical status), intraoperative outcomes, and case starts after 4 PM. The primary outcome of interest for the study was whether an incidental durotomy occurred; dural tear, CSF leak, and dural rent were considered to be synonymous with incidental durotomy.

To classify the invasiveness of the surgical procedure, we modified the surgical invasiveness score derived from the adult spinal deformity-surgical (ASD-S) score previously described by Neuman and colleagues. ${ }^{11}$ They showed that the ASD-S score outperformed the Surgical Invasiveness Index, a competing methodology for quantifying surgical invasiveness. Our modification, presented in Supplementary Table 1, includes discectomy and uses modified weighting of the surgical maneuvers.

\section{Statistical Analysis}

All data were gathered using Microsoft Excel and then analyzed using JMP (version 14, SAS Institute). Descriptive statistics constituted mean and standard deviation for continuous variables and proportions for dichotomous and categorical variables. Inferential statistics were employed using the Student t-test for continuous variables and chisquare test for categorical variables. Multivariable logistic regression with the primary outcome of incidental durotomy was used for variables that were significant on univariable analysis. The model performance was then assessed using receiver operating characteristic (ROC) curve analysis to derive the area under the curve (AUC). To evaluate for possible overfitting, bootstrapping with 1000 samples was performed to estimate optimism. The lack-of-fit test was then performed using the bootstrapped data set, with the null hypothesis being that the model does not fit the data set.

\section{Model Deployment}

To generate a clinical calculator from the results of the multivariable analysis, we used the Shiny package from R (R Foundation for Statistical Computing) and deployed the resulting calculator online.

\section{Validation of Durotomy Classification Algorithm}

As part of the study, we sought to validate a previously published natural language processing algorithm ${ }^{9}$ designed to reliably detect the occurrence of incidental durotomy from operative reports. Human review of operative reports was used as the gold-standard definition for the occurrence of intraoperative durotomy. Free text from the operative report was then fed into the natural language algorithm, which classified cases as having been complicated by an incidental durotomy or not. In short, this algorithm processed text in the operative notes to automatically recognize words of interest such as "tear" or "leak." The discrimination of the algorithm was assessed using an ROC curve with the following previously described interpretation of AUC values: $0.5-0.6=$ fail; $0.6-0.7=$ poor; $0.7-0.8=$ fair; $0.8-0.9=$ good; and $0.9-1=$ excellent. ${ }^{12}$ Additional metrics used to assess model performance were the area under the precision-recall curve (AUCPRC), the Brier score, sensitivity (recall), specificity, negative predictive value, positive predictive value (precision), F1-score, positive likelihood ratio, and negative likelihood ratio. The null model Brier score (score for an algorithm that predicts probabilities equal to the population prevalence of the outcome for every patient) was used to benchmark the algorithm Brier score.

\section{Results}

\section{Predictors of Incidental Durotomy}

On our initial screen, we identified 1353 patients, of whom 1279 met the final inclusion/exclusion criteria for the study. The overall incidental durotomy rate was $8.4 \%$ 
TABLE 1. Data for 1279 patients undergoing lumbar spine surgery

\begin{tabular}{|c|c|c|c|}
\hline Factor & $\begin{array}{l}\text { No Incidental Durotomy } \\
\qquad(\mathrm{n}=1171)\end{array}$ & $\begin{array}{l}\text { Incidental Durotomy } \\
\qquad(n=108)\end{array}$ & p Value \\
\hline Mean age, yrs & $59 \pm 15$ & $65 \pm 13$ & $<0.001$ \\
\hline Male sex & $605(51.7)$ & $56(51.9)$ & 0.98 \\
\hline Mean BMI, kg/m² & $29.7 \pm 5.9$ & $30.3 \pm 7.0$ & 0.41 \\
\hline Mean CCl & $1 \pm 2$ & $1 \pm 2$ & 0.16 \\
\hline Mean ASA class & $2 \pm 1$ & $3 \pm 1$ & 0.025 \\
\hline Revision surgery & $313(26.7)$ & $52(48.1)$ & $<0.001$ \\
\hline Mean ASD-S score* & $8.3 \pm 7.5$ & $9.7 \pm 7.8$ & 0.073 \\
\hline \multicolumn{4}{|l|}{ Instrumented levels } \\
\hline No instrumentation & $621(53.0)$ & $49(45.4)$ & \multirow{3}{*}{0.318} \\
\hline$<3$ & $257(21.9)$ & $24(22.2)$ & \\
\hline $3-6$ & $293(25.0)$ & $35(32.4)$ & \\
\hline Pelvic fixation & $81(6.9)$ & $10(9.3)$ & 0.39 \\
\hline Mean surgical duration, mins & $194.2 \pm 95.4$ & $249.8 \pm 100.3$ & $<0.001$ \\
\hline Incision time after 4 PM & $55(4.7)$ & $12(11.1)$ & 0.011 \\
\hline \multicolumn{4}{|l|}{ Specialty } \\
\hline Neurosurgery & $656(56.0)$ & $61(56.5)$ & \multirow{2}{*}{0.94} \\
\hline Orthopedics & $515(44.0)$ & $47(43.5)$ & \\
\hline Mean yrs as surgeon since training & $14.0 \pm 7.1$ & $13.3 \pm 7.5$ & 0.36 \\
\hline \multicolumn{4}{|l|}{ 1st-assist personnel } \\
\hline PGY2 & $180(15.4)$ & $18(16.7)$ & \multirow{7}{*}{0.846} \\
\hline PGY3 & $288(24.6)$ & $24(22.2)$ & \\
\hline PGY4 & $59(5.0)$ & $8(7.4)$ & \\
\hline PGY5 & $27(2.3)$ & $2(1.9)$ & \\
\hline PGY6 & $61(5.2)$ & $7(6.5)$ & \\
\hline PGY7 & $42(3.6)$ & $2(1.9)$ & \\
\hline Fellow & $514(43.9)$ & $47(43.5)$ & \\
\hline 30-day readmission & $48(4.1)$ & $6(5.6)$ & 0.49 \\
\hline Mean LOS, days & $4 \pm 3$ & $5 \pm 3$ & $<0.001$ \\
\hline
\end{tabular}

(108/1279). The demographics of these patients are presented in Table 1. Those who received incidental durotomies were significantly older (mean age $65 \pm 13$ years vs $59 \pm 15$ years, $\mathrm{p}<0.001)$ and had a worse overall ASA class (mean $3 \pm 1$ vs $2 \pm 1, p=0.025$ ). There were no significant differences in terms of sex, BMI, or CCI. The incidental durotomy group also included a greater proportion of revision surgeries (48.1\% vs $26.7 \%, \mathrm{p}<0.001)$ and had significantly longer surgeries (mean $249.8 \pm 100.3$ minutes vs $194.2 \pm 95.4$ minutes, $\mathrm{p}<0.001)$. Although the surgical invasiveness scores were higher in the incidental durotomy group (mean $9.7 \pm 7.8$ points vs $8.3 \pm 7.5$ points), this only trended toward significance $(\mathrm{p}=0.07)$. There was no significant difference regarding the number of instrumented levels $(\mathrm{p}=0.318)$. Interestingly, a significantly greater proportion of incidental durotomy cases had an initial incision time that was after 4 PM $(11.1 \%$ vs $4.7 \%, p=0.011)$. Lastly, cases with durotomies were associated with an increase of
1 day of length of stay (LOS; mean $5 \pm 3$ days vs $4 \pm 3$ days, $\mathrm{p}<0.001$ ). In the multivariable analysis (Table 2 ), the independent risk factors included surgical duration (OR 1.005 per minute, 95\% CI 1.003-1.007; p < 0.001), revision surgery (OR 2.029, 95\% CI 1.335-3.082; $\mathrm{p}=0.001)$, older age (OR 1.030 per year, 95\% CI 1.012-1.048; p < 0.001), and incision time after 4 PM (OR 3.771, 95\% CI 1.816-7.827; p $=0.001)$. When this model was then applied, it performed with fair predictive accuracy (AUC 0.73). After bootstrapping with 1000 samples, the optimism of the model was negligible (optimism $=0.003$ ). The final optimismcorrected AUC remained 0.73, and the lack-of-fit statistic was not significant ( $\mathrm{p}>0.99)$, displaying excellent model performance on the bootstrapped samples. This original predictive model was then used to create the web-based clinical risk calculator listed at the following link: https:// jhuspine3.shinyapps.io/Incidental_Durotomy_Calculator/.

External validation of the previously developed NLP 
TABLE 2. Multivariable analysis for association between potential risk factors and incidence of incidental durotomy

\begin{tabular}{lccr}
\hline \multicolumn{1}{c}{ Factor } & OR & $95 \% \mathrm{Cl}$ & $\mathrm{p}$ Value \\
\hline Surgical duration, per minute & 1.005 & $1.003-1.007$ & $<0.001$ \\
\hline Revision surgery & 2.029 & $1.335-3.082$ & $\mathbf{0 . 0 0 1}$ \\
\hline Age, per year & 1.030 & $1.012-1.048$ & $<0.001$ \\
\hline Incision time after 4 PM & 3.771 & $1.816-7.827$ & $\mathbf{0 . 0 0 1}$ \\
\hline ASA class & & & 0.792 \\
\hline
\end{tabular}

Boldface type indicates statistical significance.

algorithm in this cohort resulted in an AUC of 0.97 (95\% CI 0.95-0.99) (Fig. 1). Additionally, the model achieved an AUC-PRC of 0.81 (95\% CI 0.70-0.88) and Brier score of 0.03 (95\% CI 0.02-0.04) relative to the null model Brier score of 0.08 (Table 3 ). At a threshold of 0.05 , the algorithm had sensitivity of 0.84 , specificity of 0.96 , negative predictive value of 0.99 , positive predictive value of 0.65 , F1-score of 0.73, positive likelihood ratio of 19.7, and negative predictive value of 0.16 (Table 4).

At a threshold of 0.50 , the algorithm had sensitivity of 0.69 , specificity of 0.99 , negative predictive value of 0.97 , positive predictive value of 0.85 , F1-score of 0.77 , positive likelihood ratio of 62.6, and negative likelihood ratio of 0.31 .

\section{Discussion}

Incidental durotomy is a common complication of lumbar spine surgery that is seen in up to $11 \%$ of lumbar surgeries. ${ }^{1,2,13}$ Here, we used a large, single-institution series of patients undergoing lumbar surgery for degenerative pathologies to find independent risk factors for incidental durotomy. We found that the risk of incidental dural tear was independently associated with increased patient age, greater surgical duration, revision versus index surgery, and surgical start time after 4 PM. Surgical invasiveness was not independently predictive but approached statistical significance. The model based on these results was used to generate and deploy a clinical calculator, which was found to have fair diagnostic accuracy with an AUC of 0.73 .

\section{Impact of Durotomy on Patient Outcomes and Care Costs}

Durotomy is a known complication of spine surgery. Consequences of durotomy include postoperative headache, meningitis, new-onset neurological deficit, pseudomeningocele, and need for surgical revision. ${ }^{4}$ Durotomy can lead to worse short-term patient quality-of-life outcomes and higher levels of patient dissatisfaction. ${ }^{6}$ Strömqvist and colleagues investigated postoperative quality-of-life outcomes in a cohort of 64,431 patients who underwent surgery for lumbar stenosis, spondylolisthesis, or disc herniation. ${ }^{6}$ Patient-reported quality-of-life outcomes were evaluated using the EQ-5D, SF-36, and Oswestry Disability Index. Using these endpoints, they found that durotomy was associated with statistically significantly worse outcomes on the EQ-5D and SF-36 mental health component score. Additionally, patients who sustained incidental durotomies reported being dissatisfied with the outcomes of surgery at rates up to $50 \%$ higher than patients who did not suffer durotomies.

As a second concern, the increased hospital LOS, postoperative antibiotic requirement, and risk of requiring revision surgery all increase direct care costs. ${ }^{4}$ This both increases costs to the patient and healthcare system, consequently decreasing the cost-effectiveness of spine surgery. Using a cohort of more than 40,000 patients from the Medicare insurance database, Puvanesarajah and Hassanzadeh $^{5}$ found that expenditures for patients with incidental durotomies were more than $\$ 4000$ higher per patient, a nearly $67 \%$ increase. More recently, Alluri et al. ${ }^{3}$ reported on a 1:2 matched cohort of 26,378 patients in the PearlDiver database (a multipayer data set). Patients in the dural tear cohort had median 90-day total costs that were more than $\$ 10,000$ higher (120\% higher) than costs in the control cohort. Although neither study provided a breakdown in these cost differences, it is likely that the increased costs stem from higher rates of readmission (up to 2-fold
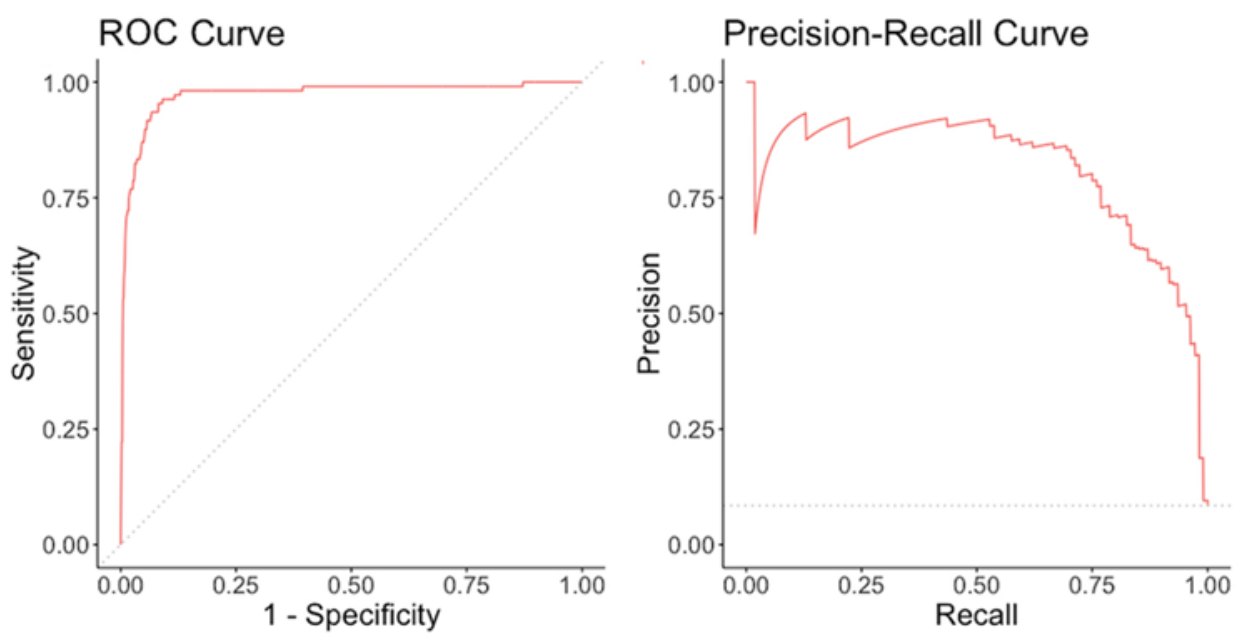

FIG. 1. ROC curve (left) and PRC (right) on external validation of NLP method to identify incidental durotomy; $n=1279$. Figure is available in color online only. 
TABLE 3. Overall performance of NLP algorithm on external validation in 1279 patients

\begin{tabular}{lc}
\hline & NLP Algorithm $(95 \% \mathrm{Cl})$ \\
\hline AUC-ROC & $0.97(0.95-0.99)$ \\
\hline AUC-PRC & $0.81(0.70-0.88)$ \\
\hline Brier score & $0.03(0.02-0.04)$ \\
\hline Calibration intercept & $1.03(0.59-1.46)$ \\
\hline Calibration slope & $0.67(0.57-0.76)$ \\
\hline
\end{tabular}

Null model Brier score $=0.08$

higher) ${ }^{3}$ and longer hospitalizations (1.5-2 days longer, on average) ${ }^{5,6}$ Furthermore, the current article found that the occurrence of an incidental durotomy led to an extra day spent in the hospital. There is a question, however, of whether increased rates of reoperation drive the greater costs. Prospective studies have failed to demonstrate an association between durotomy and increased rates of reoperation ${ }^{14}$ even though rates of persistent CSF leak have been reported to be as high as $21 \%$ in some series..$^{15}$

\section{Prior Investigations of Durotomy Risk Factors}

Multiple, large prospective $e^{7,13,16}$ and retrospective ${ }^{2}$ studies have been published describing risk factors for incidental durotomy, including several looking at independent risk factors. ${ }^{7,16}$ Together, these studies have found risk factors for durotomy to include a history of prior spine surgery, greater patient age, and larger patient body habitus. ${ }^{716}$ Similar to these prior studies, we also found revision surgery (vs index surgery) and older patient age to be associated with increased risk of incidental durotomy. Older patient age likely confers increased risk as aging of the dura is associated with a decrease in dural tensile strength. ${ }^{17,18}$ The dura of older patients is presumably more fragile and therefore more likely to rupture from manipulation. By contrast, revision surgery is complicated by dura that is adherent to the surrounding tissues secondary to increased transforming growth factor- $\beta$ expression, which causes epidural fibrosis..$^{19}$ Failure to account for and transect these adhesions during decompression of the thecal sac leads to increased tension on the thecal sac during retraction, increasing the risk for formation of a dural rent.

Additionally, we found greater surgical duration and case starts after 4 PM to be independent predictors of experiencing an incidental durotomy. The risk of increased duration has previously been shown by Desai et al. ${ }^{20}$ when this group evaluated risk factors for incidental durotomy in 799 patients undergoing discectomies in the SPORT (Spine Patient Outcomes Research Trial). This is not surprising, as greater surgical duration could indicate more technically difficult operations, leading to greater complications. The finding that later case starts (after 4 PM) for elective spine surgeries are associated with increased incidence of incidental durotomy has not been previously described. However, Halvachizadeh et al. ${ }^{21}$ analyzed how time of day affected complication rates in orthopedic trauma surgery for 31,692 patients and found that surgery performed in the afternoon and at night had increased complications compared with morning surgeries. Multiple factors have been
TABLE 4. Performance of NLP algorithm on external validation for 1279 patients

\begin{tabular}{lcc}
\hline & \multicolumn{2}{c}{ NLP Algorithm $(95 \% \mathrm{Cl})$} \\
\cline { 2 - 3 } & Threshold $=0.05$ & Threshold $=0.50$ \\
\hline Sensitivity (recall) & $0.84(0.76-0.91)$ & $0.69(0.60-0.78)$ \\
\hline Specificity & $0.96(0.94-0.97)$ & $0.99(0.98-0.99)$ \\
\hline Negative predictive value & $0.99(0.98-0.99)$ & $0.97(0.96-0.98)$ \\
\hline $\begin{array}{l}\text { Positive predictive value } \\
\text { (precision) }\end{array}$ & $0.65(0.56-0.72)$ & $0.85(0.76-0.92)$ \\
\hline F1-score & $0.73(0.64-0.80)$ & $0.77(0.67-0.84)$ \\
\hline LR+ & $19.7(14.9-26.2)$ & $62.6(35.9-109)$ \\
\hline LR- & $0.16(0.11-0.25)$ & $0.31(0.23-0.41)$ \\
\hline LR-
\end{tabular}

$\mathrm{LR}-=$ negative likelihood ratio; $\mathrm{LR+}=$ positive likelihood ratio.

hypothesized to lead to this adverse effect, including surgeon fatigue and the presence of new surgical team members unfamiliar with the procedure. ${ }^{21}$ Therefore, as elective surgeries by definition do not require emergency operations, the present results advocate against scheduling elective cases after 4 PM, especially in complex surgical cases.

\section{External Validation of NLP Algorithm}

The process of analyzing large patient data sets and electronic medical records has revolutionized how patient care is assessed and how necessary improvements are identified. However, one issue with data extraction using these sources is that much of the information is inputted as free text, rather than "clean" coding. For this reason, the use of NLP to identify outcomes in these unorganized data entries is becoming increasingly favored. ${ }^{22}$ In the field of oncology, NLP algorithms have been developed to automatically search unstructured radiology reports for both detection and progression of pathological lesions. ${ }^{23} \mathrm{In}$ the spine literature, NLP algorithms have been developed to search lumbar spine radiology reports for significant findings, such as fractures, degenerative disc disease, and stenosis. ${ }^{24}$ While both of these examples could have been performed using manual data extraction, this becomes less possible when working with large sets of data, which are quickly becoming the gold standard for research groups and hospital quality/safety departments. The present study validated the previous work of Karhade et al. ${ }^{9}$ by finding the NLP algorithm to have an AUC of 0.97 in detecting incidental durotomies in this elective lumbar spine surgery population. This novel method can be utilized by hospitals to track the incidence of this complication, as the current dependence on Current Procedure Terminology and International Classification of Diseases codes has been shown to lack in sensitivity, which can affect both reported outcomes and billing. ${ }^{9}$ Furthermore, researchers with large data sets (i.e., national spine registries) can rely on this method to most accurately identify patients with this complication, thus easing the work of manual extraction while ensuring precise data extraction.

\section{Limitations}

The present study has several limitations. First, the re- 
sults of the present study are drawn from a retrospective single-institution cohort. The retrospective nature precludes us from determining whether the risk factors identified here actually confer the increased risk of durotomy or are merely surrogates associated with the actual mitigators of risk. Yet the fact that these same risk factors have been found in other studies suggests that the relationships are real. Similarly, the single-institution nature may mean that the present results cannot be generalized to other populations. Also, this study did not analyze the effect of overlapping surgeries, which may be a risk factor due to the decreased oversight and thus could play a role in surgical complications. Furthermore, this model was assessed only using the entire training set. However, we employed a relatively large cohort of more than 1000 patients operated on by more than a dozen unique surgeons, and therefore we believe that the resulting calculator can be applied to other populations. Our results are also limited in their generalizability, as the patients examined were all undergoing elective operations for degenerative lumbar pathologies; the results may therefore not apply to other spine populations. Yet given that the identified variables are not unique to surgeries for degenerative pathologies, we believe that the calculator may be more broadly applicable. Additional research using larger, multiinstitutional cohorts is necessary to validate the present findings.

\section{Conclusions}

Using multivariable analysis, we find that increased surgical duration, greater patient age, case starts after 4 $\mathrm{PM}$, and a history of prior spine surgery are all independent positive predictors of incidental durotomy in patients undergoing elective lumbar surgery. Additionally, we put forth the first version of a clinical calculator for durotomy risk that could be used prospectively by spine surgeons when counseling patients about their surgical risk. Lastly, we present an external validation of an NLP algorithm to identify incidental durotomies. We believe that these tools can aid clinicians and researchers in their efforts to prevent this costly complication in spine surgery.

\section{References}

1. Ghobrial GM, Theofanis T, Darden BV, et al. Unintended durotomy in lumbar degenerative spinal surgery: a 10year systematic review of the literature. Neurosurg Focus. 2015;39(4):E8.

2. Iyer S, Klineberg EO, Zebala LP, et al. Dural tears in adult deformity surgery: incidence, risk factors, and outcomes. Global Spine J. 2018;8(1):25-31.

3. Alluri R, Kang HP, Bouz G, et al. The true effect of a lumbar dural tear on complications and cost. Spine (Phila Pa 1976). 2020;45(3):E155-E162.

4. Enders F, Ackemann A, Müller S, et al. Risk factors and management of incidental durotomy in lumbar interbody fusion surgery. Clin Spine Surg. 2018;31(3):127-131.

5. Puvanesarajah $\mathrm{V}$, Hassanzadeh $\mathrm{H}$. The true cost of a dural tear: medical and economic ramifications of incidental durotomy during lumbar discectomy in elderly Medicare beneficiaries. Spine (Phila Pa 1976). 2017;42(10):770-776.

6. Strömqvist F, Sigmundsson FG, Strömqvist B, et al. Incidental durotomy in degenerative lumbar spine surgery-a register study of 64,431 operations. Spine J. 2019;19(4):624-630.
7. Baker GA, Cizik AM, Bransford RJ, et al. Risk factors for unintended durotomy during spine surgery: a multivariate analysis. Spine J. 2012;12(2):121-126.

8. Buck JS, Yoon ST. The incidence of durotomy and its clinical and economic impact in primary, short-segment lumbar fusion: an analysis of 17,232 cases. Spine (Phila Pa 1976). 2015;40(18):1444-1450.

9. Karhade AV, Bongers MER, Groot OQ, et al. Natural language processing for automated detection of incidental durotomy [published online December 23, 2019]. Spine J. doi:10.1016/j.spinee.2019.12.006

10. Passias PG, Horn SR, Jalai CM, et al. Comparative analysis of perioperative complications between a multicenter prospective cervical deformity database and the Nationwide Inpatient Sample database. Spine J. 2017;17(11):1633-1640.

11. Neuman BJ, Ailon T, Scheer JK, et al. Development and validation of a novel adult spinal deformity surgical invasiveness score: analysis of 464 patients. Neurosurgery. 2018;82(6):847-853.

12. Youngstrom EA. A primer on receiver operating characteristic analysis and diagnostic efficiency statistics for pediatric psychology: we are ready to ROC. J Pediatr Psychol. 2014;39(2):204-221.

13. McMahon P, Dididze M, Levi AD. Incidental durotomy after spinal surgery: a prospective study in an academic institution. J Neurosurg Spine. 2012;17(1):30-36.

14. Desai A, Ball PA, Bekelis K, et al. SPORT: Does incidental durotomy affect longterm outcomes in cases of spinal stenosis? Neurosurgery. 2015;76(suppl 1):S57-S63.

15. Jankowitz BT, Atteberry DS, Gerszten PC, et al. Effect of fibrin glue on the prevention of persistent cerebral spinal fluid leakage after incidental durotomy during lumbar spinal surgery. Eur Spine J. 2009;18(8):1169-1174.

16. Herren C, Sobottke R, Mannion AF, et al. Incidental durotomy in decompression for lumbar spinal stenosis: incidence, risk factors and effect on outcomes in the Spine Tango registry. Eur Spine J. 2017;26(10):2483-2495.

17. Van Noort R, Martin TRP, Black MM, et al. The mechanical properties of human dura mater and the effects of storage media. Clin Phys Physiol Meas. 1981;2(3):197-203.

18. Zwirner J, Scholze M, Waddell JN, et al. Mechanical properties of human dura mater in tension-an analysis at an age range of 2 to 94 years. Sci Rep. 2019;9(1):16655.

19. Albiñana-Cunningham JN, Ripalda-Cemboráin $P$, Labiano $\mathrm{T}$, et al. Mechanical barriers and transforming growth factor beta inhibitor on epidural fibrosis in a rabbit laminectomy model. J Orthop Surg Res. 2018;13(1):72.

20. Desai A, Ball PA, Bekelis K, et al. Outcomes after incidental durotomy during first-time lumbar discectomy. J Neurosurg Spine. 2011;14(5):647-653.

21. Halvachizadeh S, Teuber H, Cinelli P, et al. Does the time of day in orthopedic trauma surgery affect mortality and complication rates? Patient Saf Surg. 2019;13:8.

22. Yim W-W, Yetisgen M, Harris WP, Kwan SW. Natural language processing in oncology: a review. JAMA Oncol. 2016;2(6):797-804.

23. Cheng LTE, Zheng J, Savova GK, Erickson BJ. Discerning tumor status from unstructured MRI reports-completeness of information in existing reports and utility of automated natural language processing. J Digit Imaging. 2010;23(2):119-132.

24. Tan WK, Hassanpour S, Heagerty PJ, et al. Comparison of natural language processing rules-based and machine-learning systems to identify lumbar spine imaging findings related to low back pain. Acad Radiol. 2018;25(11):1422-1432.

\section{Disclosures}

Joseph H. Schwab: consultant for Stryker. Daniel M. Sciubba: 
consultant for Baxter, DePuy-Synthes, Globus Medical, K2M, Medtronic, NuVasive, Stryker; and unrelated grant support from Baxter, North American Spine Society, Stryker.

\section{Author Contributions}

Conception and design: Sciubba, Ehresman, Pennington, Schwab. Acquisition of data: Ehresman, Pennington, Karhade, Huq, Medikonda, Schilling, Hersh, Lubelski. Analysis and interpretation of data: Ehresman, Karhade, Feghali, Cottrill, Westbroek. Drafting the article: Ehresman, Pennington, Karhade, Schilling, Feghali, Cottrill. Critically revising the article: Sciubba,

Ehresman, Karhade, Huq, Medikonda, Schilling, Feghali, Ahmed, Cottrill, Lubelski, Westbroek, Schwab. Reviewed submitted version of manuscript: Sciubba, Ehresman, Ahmed. Approved the final version of the manuscript on behalf of all authors: Sciubba. Statistical analysis: Ehresman, Ahmed.

\section{Supplemental Information}

Online-Only Content

Supplemental material is available with the online version of the article.

Supplementary Table 1. https://thejns.org/doi/suppl/10.3171/ 2020.2.SPINE20127.

\section{Correspondence}

Daniel M. Sciubba: Johns Hopkins University School of Medicine, Baltimore, MD.dsciubb1@jhmi.edu. 